\title{
SPH Formalism for Second Order Thermodynamics
}

\author{
Philipe Mota, Gabriel S. Denicol, and Takeshi Kodama \\ Instituto de Física, Universidade Federal do Rio de Janeiro \\ C.P. 68528, Rio de Janeiro 21945-970, RJ, Brazil
}

Received on 20 September, 2006

\begin{abstract}
The structure of the dissipative relativistic hydrodynamics is discussed. The second order thermodynamics developed by Israel, Stewart and Müller is briefly reviewed to be incorporated in the scheme of SPH formalism.
\end{abstract}

Keywords: Dissipative relativistic hydrodynamics; Second order thermodynamics

\section{INTRODUCTION}

In the early stage of ultra-relativistic heavy ion collisions it is expected emergence of a new state of nuclear matter called Quark Gluon Plasma (QGP) in which quark and gluons are in a deconfined phase as predicted by the quantum chromodynamics (QCD). Ideal hydrodynamics has been applied with success to describe this early stage, giving good quantitative and qualitative description of the observables from the experiments. Despite this fact, it is still a great mystery why we are able to apply ideal hydrodynamics to this system and still get such good results.

Although the ideal fluid description is useful to understand general features of the system, we have no reason to expect that it should give accurate quantitative results, especially in microscopic systems where large fluctuations are present such as relativistic nuclear collisions[1]. In fact, we are not certain up to what extent we can conclude from the success of ideal hydrodynamics that viscosity has no great role in the description of the QGP. That is, we are not able to say that the data are consistent with the image of ideal fluid. This is mainly because we do not know the effects of the inclusion of dissipation in relativistic hydrodynamic systems. This problem has not yet been solved for complex systems such as the QGP. So there is a great need to study this question in detail to extract more precise properties of the matter.

It is known that the inclusion of viscosity in relativistic hydrodynamics imposes a new conceptional and mathematical difficulties. Israel, Stewart and Müller had pointed out[2] that the formalism of Landau and Eckart[3] leads to non-causal propagations of density wave which are unacceptable in a relativistic theory. In this formalism, although the small deviations from equilibrium are incorporated in hydro evolution, it is assumed that the thermodynamical relations remain valid just as if the system were in equilibrium. In order to cure this problem, Israel, Stewart and Müller extend the thermodynamical relations up to the second order corrections with respect to the small deviations from local equilibrium.

The Israel-Stewart-Müller approach gives rise to causal propagation. However, the equations of motion are extremely difficult to solve, even numerically, and the several parameters introduced in the model are unknown. In this paper we give a brief introduction to this second-order hydrodynamics, trying to give a main idea of its main postulates and the difficulties that arise when we try to solve them. A schematic description of how to incorporate this scheme into a well-developped relativistic hydro code such as SPHERIO[1] is presented.

\section{PHENOMENOLOGICAL THEORY}

In this section we attempt to summarize the phenomenological derivation of the relativistic viscous hydrodynamics equations with the second order thermodynamics. Tensor notation is used in the whole article thus the equations to show the manifestly covariant nature of the system. Covariant derivatives with respect to $x^{\mu}$ are denoted by $; \mu$. We take the unit $c=1$ so that the normalization of the 4-velocity is

$$
u^{\mu} u_{\mu}=1
$$

From this, we have

$$
u^{\mu} u_{\mu ; v}=0,
$$

so that the 4-velocity is orthogonal to its derivative. This property is used frequently in this paper. It is thus useful to introduce the tensor,

$$
\Delta^{\mu \nu}:=g^{\mu \nu}-u^{\mu} u^{v}
$$

which projects, when contracted, into the transverse direction with respect to $u^{\mu}$. Using the constraint (1) we can consider only the spatial components, $u^{i}$, as independent variables. In addition to the velocity, several thermodynamcal quantities, such as baryon number density, entropy density, etc are treated as independent variables of the theory. For a density $n$ of a conserved quantity such as baryon number, we have the continuity equation,

$$
\left(n u^{\mu}\right)_{; \mu}=0
$$

as constraint.

\section{A. Equilibrium}

For ideal fluid, the fluid's entropy is conserved

$$
\left(s u^{\mu}\right)_{; \mu}=0 .
$$

In local equilibrium, the energy density then should be specified by

$$
\varepsilon(n, s)
$$


through the equation of state of the matter, relating the particle and entropy densities with the energy density. Here, for simplicity, we only consider non-zero baryon number as conserved charge.

The temperature $T(=: 1 / \beta)$ and chemical potential $\mu$ are thus defined with the aid of the first law of thermodynamics as $T:=\partial \varepsilon / \partial s$ and $\mu:=\partial \varepsilon / \partial n$. In this paper the thermodynamical potentials to be used are $\beta_{\mu}:=\beta u_{\mu}$ and $\alpha:=\mu \beta$. These quantities determine the pressure $P$ in terms of the Euler's relation,

$$
\beta \varepsilon=s-P \beta+\alpha n \text {. }
$$

Calculating the covariant derivative of (5) and using the above thermodynamical potential definitions one gets

$$
\beta \varepsilon_{; \mu}=s_{; \mu}+\alpha n_{; \mu}
$$

Combining (6) and (7) it is easy to obtain the Gibbs' relation

$$
(P \beta)_{; \mu}=-\varepsilon \beta_{; \mu}+n \alpha_{; \mu}
$$

Multiplying both sides of (6) and (8) by $u^{\mu}$ one gets the covariant Euler's equation

$$
\varepsilon \beta^{\mu}=s u^{\mu}-P \beta^{\mu}+\alpha n u^{\mu}
$$

and the covariant Gibbs' relation

$$
\left(P \beta^{\mu}\right)_{; \lambda}=-\varepsilon u^{\mu} \beta_{; \lambda}+P \beta u_{; \lambda}^{\mu}+n u^{\mu} \alpha_{; \lambda}
$$

Taking the divergence of (9) and using (10) and the conservations laws (3) and (4) it is found that

$$
\left(\varepsilon u^{\mu}\right)_{; \mu}+P u_{; \mu}^{\mu}=0
$$

With some manipulations with the help of (2) it is easy to see that this last equation introduces a new conserved quantity

$$
T_{e q ; \mu}^{\mu v}:=\left(\varepsilon u^{\mu} u^{v}-P \Delta^{\mu v}\right)_{; \mu}=0,
$$

which is known as the equilibrium energy-momentum tensor.

It is useful to rewrite (10) using the energy-momentum tensor

$$
\left(P \beta^{\mu}\right)_{; \lambda}=-T_{e q}^{\mu v} \beta_{v ; \lambda}+n u^{\mu} \alpha_{; \lambda}
$$

\section{B. Off-equilibrium}

Let us consider the phase space of the thermodynamical system, where the equilibrium is represented by a hypersurface in this space which is specified by independent variables $n, s$ and $u^{i}$. States near the equilibrim should be close to this hypersurface so that these states must be addressed by, in addition to these equilibrium variables $n, s$ and $u^{i}$, a finite set of off-equilibrium quantities that should vanish in equilibrium.

To accomodate these new quantities off-equilibrium fluxes are introduced. These must keep some relation with the equilibrium ones. For instance, the 4-velocity parallel part of the particle flux and energy-momentum tensor are kept unchanged

$$
u_{\mu} N^{\mu}=n, \quad u_{\mu} u_{v} T^{\mu v}=\varepsilon
$$

Then the off-equilibrium effects must only appear in the transverse components of these quantities with respect to $u_{\mu}$. Thus the expression for the particle flux and energy-momentum tensor can be generally written as

$$
\begin{gathered}
N^{\mu}=n u^{\mu}+\Delta_{v}^{\mu} q^{v}, \\
T^{\mu v}=\varepsilon u^{\mu} u^{v}-(P+\Pi) \Delta^{\mu v}+\mathcal{P}^{(\mu v)} \sigma \tau \pi^{\sigma \tau} .
\end{gathered}
$$

It has been emphasized in the particle flux that the new term is velocity orthogonal by the use of the projector $\Delta^{\mu \nu}$. In (15b) the last term should not only the orthogonal to the first term, but also it should be symmetric and traceless. This can be ensured by the use of the symmetric form of the bi-orthogonal traceless projector $\mathcal{P}^{\mu \nu \sigma \tau}=\Delta_{\lambda}^{\lambda} \Delta^{\mu \sigma} \Delta^{\nu \tau}-\Delta^{\mu \nu} \Delta^{\sigma \tau}$.

In contrast to the baryon number flux, the entropy flux has an additional contribution from second order terms in the longitudinal direction:

$$
u_{\mu} S^{\mu}=s+u_{\mu} Q^{\mu} .
$$

Of course, $Q^{\mu}$ must vanish once the equilibrium is achieved by the fluid.

From (6), using (14) and (16) and supposing arbitrary 4velocities one deduces

$$
\beta_{v} T^{\mu v}=S^{\mu}-P \beta^{\mu}+\alpha N^{\mu}-Q^{\mu} .
$$

The above equation differs from equilibrium for the second order term $Q^{\mu}$.

Differentiating (17) and using (10) one gets

$$
\begin{aligned}
S_{; \lambda}^{\mu}=\left(T^{\mu v}-T_{e q}^{\mu v}\right) \beta_{v ; \lambda}-\left(N^{\mu}-\right. & \left.n u^{\mu}\right) \alpha_{; \lambda}+Q_{; \lambda}^{\mu} \\
& +\beta_{v} T_{; \lambda}^{\mu v}-\alpha N_{; \lambda}^{\mu} .
\end{aligned}
$$

Taking the trace of the last expression it is straightforward to arrive at

$$
S_{; \mu}^{\mu}=\left(T^{\mu v}-T_{e q}^{\mu v}\right) \beta_{(\mu ; v)}-\left(N^{\mu}-n u^{\mu}\right) \alpha_{; \mu}+Q_{; \mu}^{\mu} .
$$

The term $Q^{\mu}{ }_{; \mu}$ is a correction to the usual[3] second order relation. This equation can also be seen as a series expansion of the entropy divergence where the first two terms vanish and we are taking up to third order terms.

With the aid of (15) the above equation can be expressed in terms of the off-equilibrium dynamic quantities

$$
S_{; \mu}^{\mu}=\left(-\Pi \Delta^{\mu v}+\pi^{\mu v}\right) \beta_{(\mu ; v)}-q^{\mu} \alpha_{; \mu}+Q_{; \mu}^{\mu} .
$$

The four-vector $Q^{\mu}$ which represents the deviation from the thermal equilibrium may not be a perfect differential, depending on the integration path of the system and its functional form can not be specified as a function of other independent thermodynamical quantities. Therefore, in order to establish the phenomenological theory, we should parametrize 
the scalar $Q^{\mu}{ }_{; \mu}$ rather than $Q^{\mu}$ in terms of our independent variables. The most general form of $Q^{\mu} ; \mu$ expressed in terms of the off-equilibrium independent dynamic quantities and involving third order terms is

$$
\begin{aligned}
Q_{; \mu}^{\mu} & =a_{0} \Pi \dot{\Pi}+a_{1} q^{\mu} \dot{q}_{\mu}+a_{2} \pi^{\mu v} \dot{\pi}_{\mu v} \\
+ & \left(b_{0} q^{\mu} q^{v}+b_{1} \pi^{\mu \lambda} \pi_{\lambda}^{v}\right) u_{(\mu ; v)}+2\left(c_{0} \Pi q^{\mu}+c_{1} q^{v} \pi_{v}^{\mu}\right) \dot{u}_{\mu} \\
& +\left(d_{0} \Pi_{; \mu}+d_{1} \pi_{\mu ; v}^{v}\right) q^{\mu}+e_{0} \Pi q_{; \mu}^{\mu}+e_{1} \pi^{\mu v} q_{(\mu ; v) .}
\end{aligned}
$$

The coefficients introduced above may all be themodynamic potentials depending on $n$ and $s$.

We further add the constraint which ensures the positivity of the entropy production

$$
S_{; \mu}^{\mu}=\zeta_{V} \Pi^{2}+\kappa q_{\mu} q^{\mu}+\zeta_{S} \pi^{\mu v} \pi_{\mu v} .
$$

The parameters $\zeta_{V}, \kappa$ and $\zeta_{S}$ are determinied by the properties of the fluid and are dinamically constant.

Equalizing (21) and (22) and using that $\Pi, q^{\mu}$ and $\pi^{\mu \nu}$ are independent variables, the expressions for the heat flux and viscous stress tensors are obtained

$$
\begin{gathered}
\zeta_{V} \Pi=-\beta u_{; \mu}^{\mu}+a_{0} \dot{\Pi}+c_{0} q^{\mu} \dot{u}_{\mu}+e_{0} q_{; \mu}^{\mu}, \\
\kappa q^{\mu}=\Delta^{\mu v}\left(-\alpha_{; v}+a_{1} \dot{q}_{v}+b_{0} q^{\lambda} u_{(v ; \lambda)}+c_{0} \Pi \dot{u}_{v}\right. \\
\left.+c_{1} \pi_{v}^{\lambda} \dot{u}_{\lambda}+d_{0} \Pi_{; v}+d_{1} \pi_{v ; \lambda}^{\lambda}\right) \\
\zeta_{S} \pi^{\mu v}=P^{(\mu v) \sigma \tau}\left(\beta_{(\sigma ; \tau)}+a_{2} \dot{\pi}_{\sigma \tau}+b_{1} \pi_{\sigma}^{\lambda} u_{(\tau ; \lambda)}\right. \\
\left.+c_{1} q_{\sigma} \dot{u}_{\tau}+e_{1} q_{(\sigma ; \tau)}\right) .
\end{gathered}
$$

\section{SMOOTHED PARTICLE HYDRODYNAMICS}

The Smoothed Particle Hydrodynamics (SPH) method has been succesfully applied in the description of the dynamic evolution of cosmological phenomena such as star explosions. This numerical method makes use of lagrangean coordinates, thus being well suited for rapid, assymetric explosive dynamics. This last feature has called the attention of the Heavy Ion Collision (HIC) researchers because SPH stands as a very adequate method to study the dynamical evolution of the early stage of HICs. One of the other advantages of this approach is that it can easily deal with event-by-event fluctuating initial conditions with complicated geometry. We will briefly introduce the bases of the SPH formalism and in the next section try to point a way to incorporate Second-Order Thermodynamics to it.

We start by an identity and with the application of two numerical approximations we reach the SPH parametrization of a given quantity

$$
\begin{aligned}
a\left(x^{i}, t\right) & =\int d^{3} r^{i} \delta^{3}\left(x^{i}-r^{i}\right) a\left(r^{i}, t\right) \\
& \approx \int d^{3} r^{i} W\left(x^{i}-r^{i}, h\right) a\left(r^{i}, t\right) \\
& \approx \sum_{p=0}^{N} A\left(r_{p}^{i}(t)\right) W\left(x^{i}-r_{p}^{i}(t), h\right)=: a_{S P H}\left(x^{i}, t\right) .
\end{aligned}
$$

The function $W$ is a normalized distribuition function that in the limit of $h \rightarrow 0$ it tends to the $\delta^{3}$ function. For computational complications it is also wished the $W$ is symmetric and have a finite support, that is, that its value goes strictly to 0 at a finite distance from the origin. This distance is idicated by the parameter $h$. We may define now the function

$$
\xi_{p}^{i}\left(x^{j}, t\right):=\frac{x^{i}-r_{p}^{i}(t)}{h},
$$

and without lost of generalization say that $W$ depends on $x^{i}$ and $t$ by the norm of $\xi_{p}^{i}$, that is, $W\left(x^{i}-r_{p}^{i}(t), h\right)=W\left(\left|\xi_{p}^{i}\right|\right)$.

To describe a physical system using SPH we should first elect a reference conserved density that would have its weights set to constants in such a way that its SPH parametrization becames the most close to the actual field

$$
\rho\left(x^{i}, t\right)=\sum_{p=0}^{N} R_{p} W\left(\left|\xi_{p}^{i}\left(x^{j}, t\right)\right|\right) .
$$

(We have abandoned the "SPH" subscript.) Based on this choice the weights of another quantity expressed in SPH would be related to the reference ones by

$$
A\left(r_{p}^{i}(t)\right)=R_{p} \frac{a\left(r_{p}^{i}(t)\right)}{\rho\left(r_{p}^{i}(t)\right)} .
$$

The most important feature of SPH now becames clear. Gradients of SPH parametrized quantities will not depend on the quantity's derivative, but on $W^{\prime}$

$$
a_{; i}=\sum_{p} R_{p} \frac{a_{p}}{\rho_{p}} \hat{\xi}_{p i} W^{\prime} .
$$

Here the hat has been use to denote a unitary vector.

In order to exploit in a more clever way the property above, one should write quantities always multiplying the reference density

$$
a_{; i}=(1 / \rho)\left((a \rho)_{; i}-a \rho_{; i}\right) .
$$

With (25) the local conservation of $\rho$ becomes

$$
\sum_{p} R_{p}\left(v_{p}^{i}-\dot{r}_{p}^{i}\right) \hat{\xi}_{p i} W^{\prime}=0 .
$$

Thus the assumption $v^{i}\left(r_{p}^{j}(t)\right)=\dot{r}_{p}^{i}(t)$ satisfies the conservation of $\rho$. By this assumption one may also notice that tha SPH "particles" do not behave as fluid elements.

\section{SECOND-ORDER THERMODYNAMICS IN SPH}

We have seen that the formalism of SPH is best applied to conservative systems since the conservation of its reference densities is ensured by construction. In the attempt to take to account dissipative effects we rewrite the SPH entropy density in a way that its weights may vary in time

$$
\begin{aligned}
& s^{*}:=\gamma: s\left(x^{i}, t\right)=\sum_{p} v_{p}(t) W\left(\left|\xi_{p}^{j}\left(x^{i}, t\right)\right|\right), \\
& n^{*}:=\gamma: n\left(x^{i}, t\right)=\sum_{p} b_{p}(t) W\left(\left|\xi_{p}^{j}\left(x^{i}, t\right)\right|\right) .
\end{aligned}
$$


(From now on we will use the definititions $\gamma:=u^{0}$ and $v^{i}:=u^{i} / u^{0}$.)

The equations to be solved numerically are

$$
\begin{gathered}
\left(n u^{\mu}+q^{\mu}\right)_{; \mu}=0 \\
\left(\varepsilon u^{\mu} u^{i}-(P+\Pi) \Delta^{\mu i}+\pi^{\mu i}\right)_{; \mu}=0, \\
\left(s u^{\mu}\right)_{; \mu}=\left(-\Pi \Delta^{\mu \nu}+\pi^{\mu \nu}\right) \beta_{\mu ; \nu}+\alpha q_{; \mu}^{\mu},
\end{gathered}
$$

plus (23).

The set of equations above sum up to 14 equations for the 14 independent variables $\vec{Y}:=\left\{n, s, v^{i}, \Pi, q^{i}, \pi^{1 i}, \pi^{22}, \pi^{23}\right\}$. With the use of the equation of state and Euler's relation, other thermodynamical variables such as $P, \alpha$ and $\beta$ are expressed in terms of these independent variables, so we get a closed system. The coefficients in viscous tensors equations (23) should be specified using some other theory such as transport equations. These coefficients were estimated in [2] for special cases. For more realistic system for the QCD matter, their expressions are not known yet.

Due to the lagrangean coordinates of the SPH method we may want to use the total (local) derivatives, $d / d t=$ $\left(1 / u^{0}\right) d / d \tau$. To do so we use the relation for any quantity A,

$$
A_{; 0}=d A / d t-v^{i} A_{; i}
$$

Using this transformation in (28a), it can be rewritten as

$$
\frac{d n^{*}}{d t}+q^{i} \frac{d v_{i}}{d t}+v^{i} \frac{d q_{i}}{d t}=\left(n^{*} g^{i j}+v^{j} q^{i}\right) v_{i ; j}+\left(v^{i} v^{j}-g^{i j}\right) q_{i ; j} .
$$

Applying the same approach to the other equations (28) and (23) we get a system of equations expressed in the matrix form as

$$
M \frac{d}{d t} \vec{Y}=(\mathbf{N} \cdot \nabla) \vec{Y}
$$

where $\mathbf{N}=\left(N_{1}, N_{2}, N_{3}\right)$ with $M$ and $N_{i}$ are 14 by 14 matrices and complicated functions of the 14 independent variables and other thermodynamical quanties. The $\nabla$ in the right-hand side represents the spatial part of the covariant derivative of the independent variable $\vec{Y}, \nabla_{i} \vec{Y}=\left(\begin{array}{llll}n_{; i} & s_{; i} & \cdots & \pi_{; i}^{23}\end{array}\right)$.
Although complicated, (29) is an ordinary first order differential equations and can be integrated in time for a given initial contidition. We may study the nature of sound wave propagation from this equation. For example, to verify if the system preserves the causality, we may analyse the linearized form of this equation and calculate the eigen value equation for the dispersion relation of the sound wave,

$$
\operatorname{det}|\omega M-\mathbf{k} \cdot \mathbf{N}|_{\vec{Y}=\vec{Y}_{0}}=0,
$$

where $\vec{Y}_{0}=\left(\begin{array}{lllll}n_{0} & s_{0} & 0 & \cdots & 0\end{array}\right)$ represents the hydrostatic background for the sound wave.

\section{DISCUSSION AND PERSPECTIVES}

In this work, we developed the formalism of the second order thermodynamics to be adapted into the smoothed hydrodynamc code presently used for the study of relativistic heavy ion collisions. We have constructed an explicit form of coulped equations. However, we note: a) Second-order thermodynamics brings in a set of coefficients, in its general form, that should be specified from other model, such as kinetic theories. For some special cases, they can be estimated, but for the strongly interacting matter, the most of these coefficients are unknown. b) 14 by 14 matrixes are extremely complicated and very hard to deal with. To avoid this problem the number of dimentions could be decreased (in $2+1$ dimentions the matrixes are 7 by 7 ) or some quantity could be neglected (such as baryon number and consequently heat flux). c) Some people study the simplified case of the second order thermodynamcis. Although Israel and Stewart proved causality only for the complete set of equations, it is not clear what is the minimal structure of relativistically causal viscoushydrodynamcis. This question should be important to understand the physical role of viscosity in the process of relativistic heavy ion collisions. For this purpose, we should study carefully (30). d) The variational principle cannot be directly applied to ensure the optimized SPH parametrization when dealing with dissipative systems.
[1] Y. Hama, T. Kodama, and O. Socolowski, Jr., Braz. J. Phys. 35, 24 (2005).

[2] W. Israel and J. M. Stewart, Annals Phys. 118, 341 (1979).
[3] L. Landau and E. M. Lifshitz, Fluid Mechanics, p. 500 AddisonWesley, Reading, Mass., 1958. 